\title{
ADVANCED PROTON IMAGING IN COMPUTED TOMOGRAPHY
}

\author{
S. Mattiazzo ${ }^{1, *}$, D. Bisello ${ }^{1,2}$, P. Giubilato ${ }^{1,2}$, D. Pantano ${ }^{1,2}$, N. Pozzobon ${ }^{1,2}$, W. Snoeys ${ }^{3}$ and J. Wyss ${ }^{2,4}$ \\ ${ }^{1}$ Dipartimento di Fisica e Astronomia, Università di Padova, Via Marzolo 8, Padova 35131, Italy \\ ${ }^{2}$ INFN, Sezione di Padova, Via Marzolo 8, Padova 35131, Italy \\ ${ }^{3}$ CERN, 1210 Geneva 23, Switzerland \\ ${ }^{4}$ DICeM, Università di Cassino e Lazio Meridionale, Via Di Biasio 43, Cassino, FR 03043, Italy
}

*Corresponding author: serena.mattiazzo@pd.infn.it

\begin{abstract}
In recent years the use of hadrons for cancer radiation treatment has grown in importance, and many facilities are currently operational or under construction worldwide. To fully exploit the therapeutic advantages offered by hadron therapy, precise body imaging for accurate beam delivery is decisive. Proton computed tomography (pCT) scanners, currently in their R\&D phase, provide the ultimate 3D imaging for hadrons treatment guidance. A key component of a pCT scanner is the detector used to track the protons, which has great impact on the scanner performances and ultimately limits its maximum speed. In this article, a novel proton-tracking detector was presented that would have higher scanning speed, better spatial resolution and lower material budget with respect to present state-of-the-art detectors, leading to enhanced performances. This advancement in performances is achieved by employing the very latest development in monolithic active pixel detectors (to build high granularity, low material budget, large area silicon detectors) and a completely new proprietary architecture (to effectively compress the data).
\end{abstract}

\section{INTRODUCTION}

In recent years, the use of energetic protons $\left({ }^{1} \mathrm{H}\right)$ and carbon ions $\left({ }^{12} \mathrm{C}\right)$ for cancer radiation treatment has grown in importance in all major developed countries ${ }^{(1)}$. Modern superconducting cyclotrons are compact machines that enable the acceleration of protons up to $250 \mathrm{MeV}$ and ${ }^{12} \mathrm{C}$ ions up to about $500 \mathrm{MeV}$ and the practical realisation of hadron therapy facilities worldwide.

The physics rationale behind the advantages of hadron therapy derives from the characteristic deposition of energy into the irradiated tissue in a very small volume at the Bragg peak, very different from the more uniform energy deposition achieved with $\mathrm{X}$-ray irradiation. The depth of the Bragg peak, i.e. the position of the small volume where most of energy is deposited in the body, strongly depends on the energy of the particle and on the density of the traversed tissues. As the particle energy can be controlled with a precision better than $0.5 \%{ }^{(2)}$, the precise knowledge of the tissue density along the particle path becomes the limiting factor in precisely aiming at the tumour cells and is hence critical for effectively exploiting the intrinsic hadron energy deposition resolution. Such knowledge is nowadays gathered by using standard X-ray computed tomography (X-ray CT), to generate a $3 \mathrm{D}$ image of the body target. X-ray imaging is perfect for X-ray radiotherapy, as the same particles (photons) are used for both imaging and treatment, while the precision falls short when applied to hadron therapy ${ }^{(3)}$, as hadrons interact with matter differently from photons. In practice, $\mathrm{X}$ rays do not precisely highlight the densities of the tissues, which are of upmost importance in calculating the exact energy that protons must have in order to stop in the tumour volume. This difference can lead to large errors in the positioning of the Bragg peak, which can be up to few centimetres in certain region of the head $^{(4)}$, spoiling the potential accuracy of the technique.

Among the many solutions proposed to overcome this limitation, the most promising one uses protons also for the 3D imaging of the target, actually realising a proton computed tomography, $p C T^{(2,5)}$. By tracking protons of high energy (at least $200 \mathrm{MeV}$, so they do not stop inside the body), it is possible to generate a $3 \mathrm{D}$ view of the target, exactly as in an X-ray CT, by moving the proton source around the target (or by rotating the target itself) and recording at each step the paths and energy loss of the particle. While spatial resolution is lower than that of X-ray CT (as protons undergo multiple Coulomb scattering), the density resolution is far superior, allowing for much more precise tuning of the energies of hadrons used for the radiation treatments ${ }^{(6)}$. Besides the higher precision for hadron therapy guidance, this approach has the advantage of exploiting the same accelerating machine and beam-line used for the treatment, as well as the same gantry to hold the patient. In addition, mathematical considerations and first prototype testing indicate that about $10^{9}$ proton tracks in total are necessary for a precise $3 \mathrm{D}$ reconstruction of the target, equivalent to a dose of $1.5 \mathrm{mGy}^{(5)}$. This compares very favourably to the dose necessary for a usual X-ray CT, which varies in the 15-100 mGy range depending on the target ${ }^{(7)}$.

\section{PROTON TRACKER: STATE OF THE ART}

A typical pCT R\&D set-up (Figure 1) is composed of a tracking system (to measure the proton entry and 


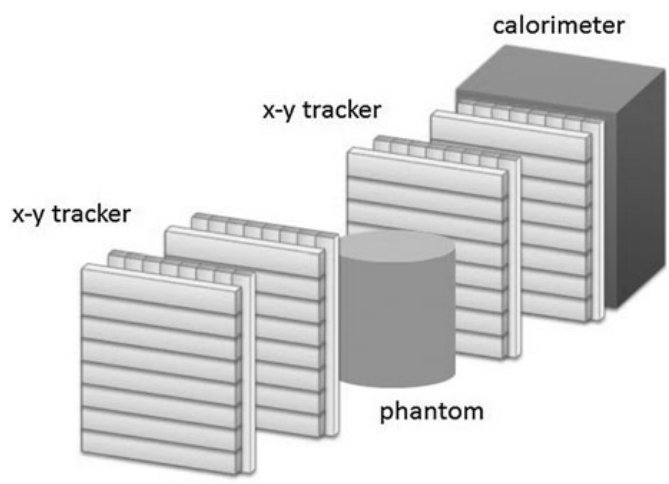

Figure 1. Layout of a typical pCT set-up. A proton enters from the left; its entry position and direction are tracked in the entrance telescope. The proton passes the phantom (patient); exit position and direction are tracked again at the exit, just before reaching the calorimeter, where the residual energy is measured.

exit directions) and a calorimeter (to measure the proton residual energy, the incoming energy being supposed to be known 'a priori').

A key aspect of the set-up is the proton-tracking detector, which requires high precision (some tens of microns resolution) and a high tracking speed (megahertz particle rate) over a large area (about $10 \times 30 \mathrm{~cm}^{2}$ ). Radiation hardness $(>1 \mathrm{kGy})$ is a further requirement for any practical device. Nearly all proposed systems use silicon strip detectors (SSDs) derived from highenergy physics experiments as tracking planes. SSDs contain an array of parallel long strip-shaped detecting diodes and return the position in one dimension of a traversing particle with around hundred micron precision and high speed (up to many megahertz, depending on the strip length) ${ }^{(5)}$. To retrieve the $x$ and $y$ position of the particles, it is necessary to use two orthogonally placed SSDs for the tracking planes for both the entrance and exit sides of the scanner. Besides introducing non-negligible amounts of material into the path of the proton (thus increasing scattering), all these systems require that no more than one particle pass through the tracking detector during an acquisition frame: multiple hits in a single frame would make particle positions reconstruction ambiguous (Figure 2).

The single particle per frame limit and the Poisson statistic dictate that for full (better than $99 \%$ ) tracking efficiency, the particle rate must be a factor of $\sim 10$ lower than the detector frame rate, e.g. $100 \mathrm{kHz}$ for a $1 \mathrm{MHz}$ refresh rate. This means that the best present set-ups, with frame rates of the order of $10 \mathrm{MHz}$, would take about $10-16$ min to record the $10^{9}$ tracks $^{(5)}$ necessary to reconstruct a complete image.

The two key weaknesses in the double-layer detector approach are the intrinsic SSD readout speed limit (due to the strip capacitance) and, more importantly,
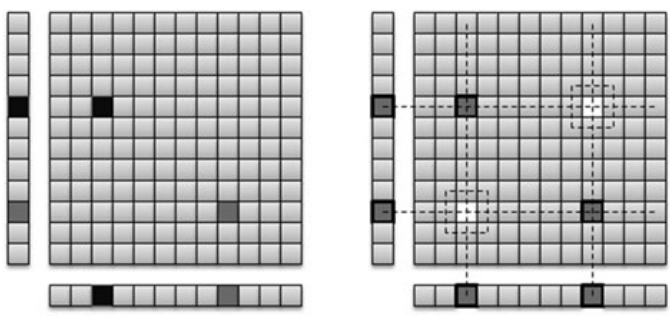

Figure 2. Traditional projection scheme. Assuming nondistinguishable signals, it is not possible to correctly reconstruct the original hits from the 'aliases' generated by the multiple hits in every projection (right).

the $x$ and $y$ projective scheme, which limits the speed/ efficiency.

A possible improvement with respect to SSDs could be a pixel detector matrix, which provides both the $x$ and $y$ coordinates on a single sensor layer. The drawback of this approach is the number of pixels to be read, $N^{2}$, compared with $2 \mathrm{~N}$ of an SSD for an equivalent resolution, which would make the data throughput unmanageable for the target speed requirements. An intelligent readout architecture, able to send off from chip only the information on fired pixels, should be therefore envisaged. One current example of pixel detector for medical imaging is the very successful Medipix family ${ }^{(8)}$, based on hybrid pixels. A hybridpixel detector combines two distinct layers: one containing the actual sensitive elements, and the other providing an advanced 'per-pixel' readout circuitry, which embeds tailored functions. For tracking purposes, where frame rate is paramount, the in-pixel circuitry should flag for reading out only those pixels actually carrying a signal, thereby drastically reducing the data output from the detector. The main limitation of hybrid-pixel detectors for practical applications in pCT scanners is the high production and assembly cost, mostly due to the bump-bonding used to connect the two layers, which also negatively affects the multiple scattering.

\section{THE PROXY DETECTOR}

To provide a global performance improvement, the detector technology and architecture have to be changed with respect to present solutions. Hence, a pixel detector integrating the strength of both SSDs and hybrid pixels in a single device capable of higher speed and resolution with a lower material budget than present systems was proposed. Such performance improvements are made possible by fabricating monolithic pixel detectors in the present-day commercial complementary metal oxide semiconductor (CMOS) technology, allowing a reduction in volume production costs compared with present, custom-made detectors. This novel tracking sensor, 


\section{S. MATTIAZZO ETAL}

dubbed ProXY (Protons $\mathrm{X}$ and $\mathrm{Y}$ ) would be based on the OrthoPix architecture, a readout scheme specially developed for tracking particles at high rates over a large $\operatorname{area}^{(9)}$. Different from hybrid sensors, this monolithic sensor would integrate sensor and readout in one piece of silicon, and therefore favourably compare with hybrid detectors in terms of assembly and production cost, and material budget.

\section{The OrthoPix architecture}

The most innovative feature of the ProXY detector is the OrthoPix architecture upon which it is based ${ }^{(9)}$. The term architecture actually refers to the way the single pixels are connected and read to generate the detector output. The OrthoPix architecture is an evolution of the traditional projection scheme used in present pCT scanners employing SSD or scintillating fibres sensors, designed to solve the difficulty of distinguishing more than one hit per frame.

The traditional projective sparsification (i.e. compression) scheme has been widely employed due to its effectiveness and simplicity of implementation. Assuming an array of data elements composed by $N^{2}$ cells, to determine the position of one cell carrying a signal, it is enough to process $2 \mathrm{~N}$ elements, thus achieving a compression ratio of $N / 2$. Using more than two projections helps disentangling multiple hits situations. The idea has been already explored in the past both in gaseous and solid-state detectors, mostly by adding a third, usually diagonal projection (Figure 3 ). The OrthoPix architecture generalises this concept to an arbitrary number of projections of arbitrary shape,

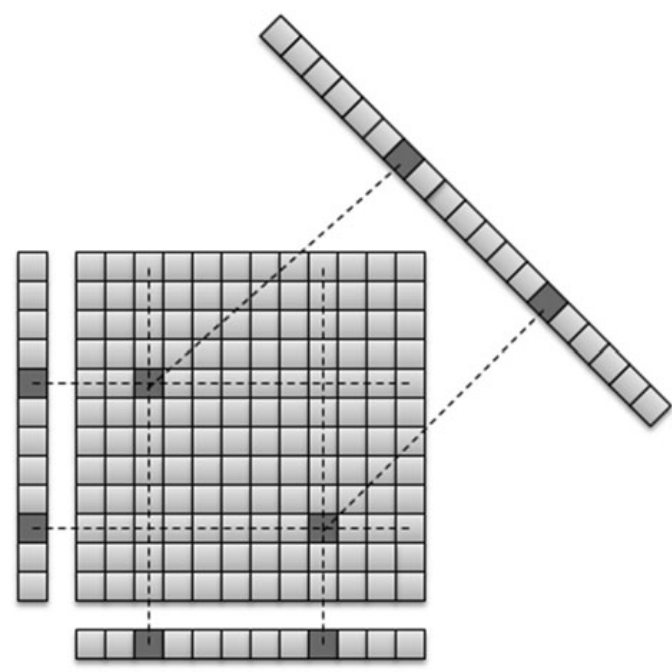

Figure 3. The additional information provided by adding a third projection, along a diagonal in this example, makes it possible to correctly reconstruct the position of more than one hit in a frame. providing a quantitative description of the achievable hits rates and efficiency for every possible configuration.

To keep the material budget low (to minimise protons scattering) and to reduce assembly cost, complexity and border effects, large base modules are necessary. The possibility to use stitching techniques to produce chips as big as the entire wafer size in modern CMOS processes allows one to realise pixel detectors of up to about $100 \mathrm{~cm}^{2}$. A four-projection OrthoPix architecture applied to a square detector leads to a compression ratio equal to $N / 4$ : maximising the size hence maximises also the data efficiency of the system. To cover the full area (about $10 \times 30$ $\mathrm{cm}^{2}$ ), a few modules will need to be tiled.

A promising starting point encompasses using square base modules of 4 or $8 \mathrm{~cm}$ side. The resolution goal of $10 \mu \mathrm{m}$ and technical considerations derived by test results on some prototypes ${ }^{(10)}$ both push for a pixel size around $20 \mu \mathrm{m}$. Tables 1 and 2 summarise the expected (simulated) maximum particle rate for such devices $\left(4 \times 4\right.$ and $8 \times 8 \mathrm{~cm}^{2}$ size $)$ using the OrthoPix architecture and a refresh rate of $50 \mathrm{MHz}(\mathrm{a}$ very reasonable speed for pixel detectors), for two different efficiencies ( 99.5 and $85 \%$ ).

Table 1 clearly shows the potential of the OrthoPix architecture in improving the performances of pCT scanner tracking detectors: a base unit sensor of

Table 1. Maximum particle rates for different module sizes $(2048 \times 2048$ and $4096 \times 4096)$, assuming the average cluster size being 4 pixels and the particle uniformity distributed across the frame.

\begin{tabular}{lccccc}
\hline $\begin{array}{l}\text { Pixel } \\
\text { count }\end{array}$ & $\begin{array}{c}\text { Pixel } \\
\text { pitch } \\
(\mu \mathrm{m})\end{array}$ & $\begin{array}{c}\text { Frame } \\
\text { size } \\
\left(\mathrm{mm}^{2}\right)\end{array}$ & $\begin{array}{c}\text { Compression } \\
\text { ratio }\end{array}$ & $\begin{array}{c}p \mathrm{~cm}^{-2} \\
(\mathrm{MHz})\end{array}$ & $\begin{array}{c}p \text { per } \\
\text { Frame } \\
(\mathrm{GHz})\end{array}$ \\
\hline 2048 & 20 & $41 \times 41$ & $512: 1$ & $\sim 120$ & $\sim 2$ \\
4096 & 20 & $82 \times 82$ & $1024: 1$ & $\sim 60$ & $\sim 4$ \\
\hline
\end{tabular}

A readout speed of $50 \mathrm{MHz}$ and an efficiency of $99.5 \%$ have been assumed.

Table 2. Maximum particle rates for different module sizes $(2048 \times 2048$ and $4096 \times 4096)$, assuming the average cluster size being 4 pixels and the particle uniformity distributed across the frame.

\begin{tabular}{lccccc}
\hline $\begin{array}{l}\text { Pixel } \\
\text { count }\end{array}$ & $\begin{array}{c}\text { Pixel } \\
\text { pitch } \\
(\mu \mathrm{m})\end{array}$ & $\begin{array}{c}\text { Frame } \\
\text { size } \\
\left(\mathrm{mm}^{2}\right)\end{array}$ & $\begin{array}{c}\text { Compression } \\
\text { ratio }\end{array}$ & $\begin{array}{c}p \mathrm{~cm}^{-2} \\
(\mathrm{MHz})\end{array}$ & $\begin{array}{c}p \text { per } \\
\text { Frame } \\
(\mathrm{GHz})\end{array}$ \\
\hline 2048 & 20 & $41 \times 41$ & $512: 1$ & $\sim 250$ & $\sim 4$ \\
4096 & 20 & $82 \times 82$ & $1024: 1$ & $\sim 104$ & $\sim 7$ \\
\hline
\end{tabular}

A readout speed of $50 \mathrm{MHz}$ and an efficiency of $85 \%$ have been assumed. 


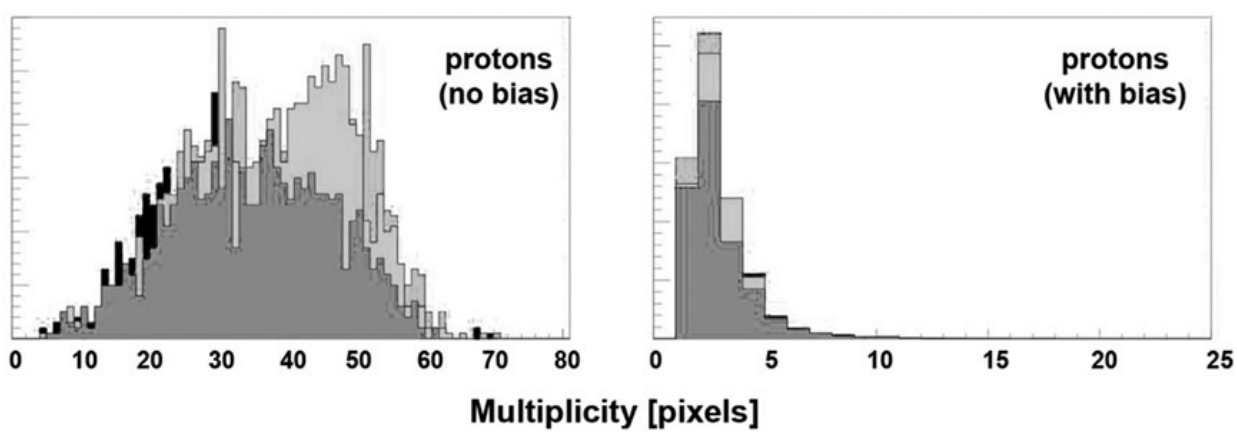

Figure 4. Cluster size for protons with no reverse bias (left) and $V_{\text {bias }}=60 \mathrm{~V}$ (right). Applying a reverse bias significantly shrinks the cluster size.

$8 \times 8 \mathrm{~cm}^{2}$ composed by $4096 \times 409620 \mu \mathrm{m}$ pitch pixels could handle alone a particle rate of $4 \mathrm{GHz}$ $\left(\approx 60 \mathrm{MHz} \mathrm{cm}^{-2}\right)$. A full detector built by tiling eight such base modules would cover an area of $8 \times 32 \mathrm{~cm}^{2}$, handling a global rate of more than $16 \mathrm{GHz}$ at full efficiency. Particle uniformity considerations anyway suggest considering as maximum rates that of a single base module: assuming a global sustainable rate of $1 \mathrm{GHz}$ at $99.5 \%$ efficiency, the total time for a complete $3 \mathrm{D}$ scan $\left(10^{9}\right.$ protons) would shrink down to about $1 \mathrm{~s}$. At lower efficiencies (hence higher dose to the patient), speed could be further increased, as shown in Table 2.

\section{Technological implementation}

To successfully implement the proposed architecture using metal connections, a deep submicron CMOS process would be used to provide sufficient metal line density for the planned large modules $(8 \mathrm{~cm})$.

Fabricating the CMOS process on a high-resistivity substrate or on a wafer with a high-resistivity epitaxial layer of a few tens of microns thick would ensure large signals with relatively low bias voltages, charge collection by drift and smaller cluster size (if compared with charge collection by diffusion).

A small prototype (a matrix of $32 \times 32$ analogue pixels) in a $90-\mathrm{nm}$ CMOS process over a $500 \Omega \mathrm{cm}^{-1}$ substrate has been already designed and produced. It was tested with different radiation sources, including $300 \mathrm{MeV}$ protons ${ }^{(10)}$. Figure 4 illustrates the variation in the cluster size with no bias and with $V_{\text {bias }}=60 \mathrm{~V}$. Clusters were reconstructed applying a double-threshold method on the matrix of pixels around a selected cluster seed: a higher threshold in the signal-to-noise ratio for the seed and a smaller one for the neighbour pixels. The histograms clearly show how applying a reverse bias significantly reduces the cluster size (and consequently the amount of data to be sent off chip).

After the successful technology evaluation phase, complete OrthoPix chips have been submitted and are now ready for testing.

\section{CONCLUSIONS}

In this article, the use of a novel tracking sensor for proton imaging in CT is presented. This sensor would have higher scanning speed (down to few seconds), better spatial resolution (down to $10 \mu \mathrm{m}$ ) and lower material budget with respect to present state-of-the-art detectors. Such advancement in performance can be achieved by employing the latest development in monolithic active pixel detectors and a completely new architecture. Together with the performance improvement, a cost reduction would result as well, as the proposed detector is based on commercially available technology.

To fully exploit the potential of the proposed tracker, such an improvement should be matched by the other parts of the scanner, in particular the calorimeter, the reconstruction computing node and the supporting scanning mechanics.

\section{REFERENCES}

1. Paganetti, H. and Bortfeld, T. Proton beam radiotherapythe state of the art. Med. Phys. 32, 2048-2049 (2005).

2. Sadrozinski, H. F. W. et al. Toward proton computed tomography. IEEE Trans. Nucl. Sci. 51, 3-9 (2004).

3. Depauw, N. and Seco, J. Sensitivity study of proton radiography and comparison with $k V$ and $M V X$-ray imaging using GEANT4 Monte Carlo simulations. Phys. Med. Biol. 56, 2407-2421 (2011).

4. Schneider, U. and Pedroni, E. Proton radiography as a tool for quality in proton therapy. Med. Phys. 22, 353-363 (1995).

5. Sadrozinski, H. F. W., Johnson, R. P., Macafee, S., Plumb, A., Steinberg, D., Zatserklyaniy, A., Bashkirov, V. A., Hurley, R. F. and Schulte, R. W. Development of a head scanner for proton CT. Nucl. Inst. Meth. A. 699, 205-210 (2013).

6. Ryu, H., Song, E. and Lee, J. Density and spatial resolutions of proton radiography using a range modulation technique. Phys. Med. Biol. 53, 5461-5469 (2008).

7. Hall, E. J. and Brenner, D. J. Cancer risks from diagnostic radiology. Br. J. Radiol. 81, 362-378 (2008).

8. Campbell, M. on behalf of the Medipix2 Collaboration. 10 years of the Medipix2 Collaboration. Nucl. Inst. Meth. A. 633, S1-S10 (2011). 


\section{S. MATTIAZZO ETAL.}

9. Giubilato, P. and Snoeys, W. OrthoPix: a novel compression architecture for pixel detectors. In: Proceedings of NSS/MIC. Anaheim, CA, 2012. DOI: 10.1109/ NSSMIC.2012.6551407.

10. Giubilato, P. et al. Monolithic pixels on moderate resistivity substrate and sparsifying readout architecture. Nucl. Instr. Meth. A. 731, 146-153 (2013).
11. Mattiazzo, S. et al. LePIX: first results from a novel monolithic pixels sensor. Nucl. Instr. Meth. A. 718, 288-291 (2013).

12. Senyukov, S., Baudot, J., Besson, A., Claus, G., Cousin, L., Dorokhov, A. and Dulinski, W. Charged particle detection performances of CMOS pixel sensor produced in a 0.18 $\mu \mathrm{m}$ process with a high resistivity epitaxial layer. Nucl. Instr. Meth. A. 730, 115-118 (2013). 